\title{
Experimental validation of an analytical kinetic model for Edge-Localized Modes in JET-ITER-Like Wall
}

\author{
C. Guillemaut ${ }^{1,2}$, C. Metzger ${ }^{1,3}$, D. Moulton ${ }^{4}$, K. Heinola ${ }^{5}$, M. O'Mullane ${ }^{6}$, \\ I. Balboa ${ }^{4}$, J. Boom ${ }^{7}$, G.F. Matthews ${ }^{4}$, S. Silburn ${ }^{4}$, E.R. Solano and JET contributors* \\ ${ }^{1}$ EUROfusion Consortium, JET, Culham Science Centre, Abingdon, OX14 3DB, UK \\ ${ }^{2}$ Instituto de Plasmas e Fusão Nuclear, Instituto Superior Técnico, Universidade Lisboa, PT \\ ${ }^{3}$ Ambassade de France à Londres, 21 Cromwell Road, London SW7 2EN, UK \\ ${ }^{4}$ CCFE, Culham Science Centre, Abingdon OX14 3DB, UK \\ ${ }^{5}$ Department of Physics, University of Helsinki, P.O. Box 43, 00560 Helsinki, Finland \\ ${ }^{6}$ Department of Physics, University of Strathclyde, Glasgow G4 ONG, UK \\ ${ }^{7}$ Max-Planck-Institut fur Plasmaphysik, Boltzmannstr. 2, D-85748 Garching, Germany \\ ${ }^{8}$ Laboratorio Nacional de Fusión, CIEMAT, 28040 Madrid, Spain \\ *See the author list of "Litaudon et al, Nucl. Fusion 57 (2017) 102001" \\ Contact Email: christophe.quillemaut@ukaea.uk or cguillemaut@ipfn.ist.utl.pt
}

\begin{abstract}
The design and operation of future fusion devices relying on $\mathrm{H}$-mode plasmas requires reliable modelling of Edge-Localized Modes (ELMs) for precise prediction of divertor target conditions. An extensive experimental validation of simple analytical predictions of the time evolution of target plasma loads during ELMs has been carried out here in more than $70 \mathrm{JET}$-ITER-Like Wall H-mode experiments with a wide range of conditions. Comparisons of these analytical predictions with diagnostic measurements of target ion flux density, power density, impact energy and electron temperature during ELMs are presented in this paper and show excellent agreement. The analytical predictions tested here are made with the "Free-Streaming" kinetic model (FSM) which describes ELMs as a quasi-neutral plasma bunch expanding along the magnetic field lines into the Scrape-Off Layer without collisions. Consequences of the FSM on energy reflection and deposition on divertor targets during ELMs are also discussed.
\end{abstract}

\section{Introduction}

Predictions of target conditions such as power density, particle flux density and impact energy during Edge-Localized Modes (ELMs) in future fusion devices relying on $\mathrm{H}$-mode plasmas is essential for the design of plasma facing components (PFC) and operational scenarios. Plasma-wall interaction issues like phase transition of the PFC material, erosion or impurity sputtering, are expected to be dominantly due to ELMs $[1,2]$. In this context, a reliable model allowing precise predictions of target conditions during ELMs to assess these phenomena would be very useful.

The "Free-Streaming" kinetic model (FSM) describes ELMs in 1-D as a plasma bunch expanding in vacuum along the magnetic field lines [3-5]. The model is based on the assumptions that ELMs are compact and conserve quasi-neutrality during their collisionless parallel transport from pedestal to targets. To satisfy these assumptions, electrons should transfer most of their energy to the ions much before the ELM filaments reach the target. As a consequence, the impact energy of the ions 
at the target should be proportional to the pedestal temperature [2]. The FSM allows analytical calculations of the time evolution of target plasma loads during ELMs and could be a powerful predictive tool.

To date, comparisons between FSM calculations of target power load or ion impact energy $\left(E_{i}\right)$ with experimental measurements have been attempted on a very limited number of Type-I ELMy H-mode discharges and were successful in ASDEXUpgrade and JET $[2,4,6]$. In order to confirm with confidence the suspicion that key aspects of ELM physics are indeed captured by the FSM, a systematic and extensive validation effort has been carried out in JET-ITER-Like Wall (ILW) H-mode experiments and is presented in this paper. A data set comprising 74 steady Type-I or Type-III ELMy $\mathrm{H}$-mode periods in JET-ILW discharges with a very wide range of input power $\left(P_{i n}\right)$, ELM frequency (Fig. 1), plasma current $\left(I_{P}\right)$, toroidal field $\left(B_{T}\right)$ and pedestal conditions have been used. These discharges were achieved with deuterium (D) or hydrogen as main species and a few of them involved nitrogen or neon seeding, see Annex Section. The configuration in use in this series of experiments features a vertical inner target and a horizontal outer target (OT). The study presented here is focused on the OT which has an excellent diagnostic coverage, see Fig. 2.

FSM predictions for the time evolution of target ion flux and power densities are tested for Type-I and Type-III ELMs in two JET-ILW H-mode cases in the next Section. The consequences of FSM physics on target electron and ion impact energy are discussed in Section 3 and 4, respectively. Finally, before concluding, the possible effects of energy reflection on target measurements during ELMs are discussed in the last Section 5.

\section{Time evolution of target plasma loads during Type-I and Type-III ELMs}

In the FSM, it is considered that the ELM filaments ejected upstream have a Maxwellian distribution of energy in both parallel and perpendicular directions. Since ELMy ions with the highest parallel energy free-stream faster to the targets than those with lower energy, it generates a characteristic time distribution of the ion and energy fluxes on the divertor targets. In a 1-D approach, ignoring potential cross-field transport effects, the strike-point surface ion flux $\left(\Gamma_{\perp}\right.$ in A.m $\left.{ }^{-2}\right)$ and power $\left(q_{\perp}\right.$ in W.m ${ }^{2}$ ) densities predicted by the FSM [5] are respectively such that:

$$
\begin{aligned}
& \Gamma_{\perp}=\Gamma_{0}+\sin \left(\theta_{\perp}\right) \frac{e n_{e}^{\text {ped }}}{c_{s}} \frac{L_{l /} L_{E L M}}{t^{2}} \exp \left(\frac{L_{l /}^{2}}{2 t^{2} c_{s}^{2}}\right) \\
& \text { and } q_{\perp}=q_{0}+\Gamma_{\perp} T_{e}^{\text {ped }}\left[\left(\frac{L_{/ /}}{c_{s}}\right)^{2} \frac{1}{t^{2}}+1\right],
\end{aligned}
$$


with the background ion flux density $\Gamma_{0}$, the background power density $q_{0}$, the target field line angle $\theta_{\perp} \approx 2-3^{\circ}$, the pedestal electron density $n_{e}^{\text {ped }}$ in $\mathrm{m}^{-3}$, the pedestal electron temperature $T_{e}^{\text {ped }}$ in $\mathrm{eV}$, the sound speed $c_{s}=\left(2 e T_{e}^{\text {ped }} / m_{i}\right)^{1 / 2}$, the ion mass $m_{i}$ in $\mathrm{kg}$, the time $t$ in $\mathrm{s}$, the target to target parallel connection length $L_{/ /}$in $\mathrm{m}$, the initial parallel extension of the ELM filaments $L_{E L M}$ in $\mathrm{m}$ and $e=1.6 \times 10^{-19} \mathrm{~J} \mathrm{eV}^{-1}$. Here, the pedestal ion temperature is assumed to be equal to $T_{e}^{\text {ped }}$.

Fast divertor Langmuir probe (LP) and Infrared thermography (IR) measurements (Fig. 2) with a time resolution of $10 \mu \mathrm{s}$ for $\Gamma_{\perp}$ and $200 \mu \mathrm{s}$ for $q_{\perp}$, respectively, were available on the OT for the 74 cases studied here. High resolution $\Gamma_{\perp}$ measurements were obtained during saturation of the ion current with LPS sweeping their voltage at $\sim 500 \mathrm{~Hz}$. Since ion saturation lasts for over $\sim 1 \mathrm{~ms}$ per $\sim 2$ ms sweeps when the voltage is typically between $\sim-150 \mathrm{~V}$ and $\sim-70 \mathrm{~V}$, full or partial Type-I ELM $\Gamma_{\perp}$ signatures are often captured and many Type-III ELMs can be observed. A coherent averaging method [7] using Beryllium II spectroscopy (Fig. 2) as an ELM marker has been used to obtain a typical average ELM time trace for each case. Both $\Gamma_{\perp}$ and $q_{\perp}$ signals are measured at the position on Tile 5 corresponding to the maximum particle flux (see Fig. 2) and thus follow the outer strike point movement.

For convenience, detailed comparison of $\Gamma_{\perp}$ and $q_{\perp}$ experimental measurements with FSM calculations has been focused on two cases: discharge \#84700 for typical large amplitude and slow Type-I ELMs with a frequency $\left(f_{E L M}\right)$ of $\approx$ $50 \mathrm{~Hz}$ (Fig. 3a and c) and discharge \#87588 for small amplitude and fast Type-III ELMs with $f_{E L M} \approx 1200 \mathrm{~Hz}$ (Fig. $3 \mathrm{~b}$ and d). Fig. $3 \mathrm{e}, \mathrm{f}, \mathrm{g}$ and $\mathrm{h}$ are discussed later on in this paper. Both parameters $L_{/ /}$and $L_{E L M}$ shown in Table 1 were adjusted in (1) and (2) to allow the best fit possible of the experimental $\Gamma_{\perp}$ and $q_{\perp}$ time traces. Mention should be made that the same $L_{/ /}$and $L_{E L M}$ values have been used in (1) and (2) and that the ELM compactness condition $L_{/ /} \gg L_{E L M}$ for FSM validity is verified here. For the Type-I ELMs of \#84700, $L_{E L M}$ can be used to estimate the ELM energy ( $E_{E L M}$ in J) such that:

$$
E_{E L M} \approx 3 N_{f i l} A_{\perp} \sqrt{2 \pi} n_{e}^{\text {ped }} L_{E L M} e T_{e}^{\text {ped }}
$$

and verify its consistency with experiment. With the number of ELM filaments $N_{f i} \approx$ $10[8,9]$ and the filament cross-section $A_{\perp} \approx 3 \times 10^{-3} \mathrm{~m}^{2}[5,10], E_{E L M} \approx 200 \mathrm{~kJ}$ which corresponds to the average variation of stored energy $(\Delta W)$ in this discharge. In \#87588, we can estimate $E_{E L M} \sim 1 \mathrm{~kJ}$ which is expected for small and fast Type-III ELMs. Unfortunately, the time resolution of $\Delta W$ measurements is not high enough to distinguish small periodic variations of this level.

The inter-ELM $L / /$ in JET-ILW is $\sim 100 \mathrm{~m}$ which is close to the fitting parameter used for Type-III ELMs in \#87588. However, $L_{/ /}$must be more than $\sim 8$ times longer to allow a fit of the Type-I ELM time traces in Fig. 3a and c for \#84700. During Type-I ELMs, perturbed $L_{/ / /}$of the order of $\sim 5-10$ times longer than the unperturbed interELM value is already suggested by ELM simulations with the JOREK fluid code 
$[11,12]$. Similar fit attempt of a $q_{\perp}$ time trace from IR during Type-I ELMs in a JETCarbon experiment also required significantly longer $L_{/ / /}$[4]. The JOREK code suggests that stochastisation of the magnetic field lines during Type-I ELM perturbations generate much longer paths for the ELMy ions than during inter-ELM. Since $L_{E L M}$ is around $\approx 210 \mathrm{~m}$ in the large Type-I ELMs of \#84700 compared to only $\approx 5 \mathrm{~m}$ in the small Type-III ELMs of \#87588, it can be suspected that the increase of $L / /$ during an ELM depends on its size and the magnetic perturbation associated with it. The perturbation may simply be too small during Type-III ELMs to affect $L_{\text {/I }}$ significantly.

Since the fits for $\Gamma_{\perp}$ and $q_{\perp}$ during Type-III ELMs in Fig. $3 \mathrm{~b}$ and d involve $\Gamma_{0}$ $=700 \mathrm{~A} \cdot \mathrm{m}^{-2}$ and $q_{0}=1 \mathrm{MW} \cdot \mathrm{m}^{-2}$ respectively, it suggests that the coherently averaged signals for these quantities are built on top of a non-negligible background in these conditions. This is consistent with the peak values being only a small factor above the inter-ELM level. The background is negligible for the fit of the coherently averaged Type-I ELM signals.

A dip can be observed in both cases for $\Gamma_{\perp}$ in Fig. $3 a$ and $b$ and is likely to be due to a small population of very high energy electrons capable of escaping the pedestal at the very beginning of the ELM. Such particles would not be repelled by the LPs at the target because of insufficient bias voltage and would decrease momentarily the measured ion flux. Such effect is not described by the FSM and is beyond the scope of the present study which focuses on the physics involved during the main ELM event.

\section{Target electron energy during Type-I and Type-III ELMs}

In Fig. $3 a$ and $b$, the LP signals have been compared to $D_{\alpha}$ line emission from $D$ recycling or desorption at the strike point calibrated with the number of ionization per Balmer photon $(\mathrm{S} / \mathrm{XB})$. These coherently averaged ionization rate densities have been obtained from $D_{\alpha}$ measurements accounting for the target electron density $\left(n_{e}\right)$ and temperature $\left(T_{e}\right)$ dependence of $\mathrm{S} / \mathrm{XB}$ as given by the Atomic Data and Analysis Structure (ADAS) [13], see Fig. 4. If we assume that the increase of $n_{e}$ during ELMs is proportional to $\Gamma_{\perp}, n_{e}$ ranges from $\approx 2$ to $\approx 5.3 \times 10^{19} \mathrm{~m}^{-3}$ in $\# 84700$ and $\mathrm{S} / \mathrm{XB}$ can be approximated by $\approx 3.33 \times 10^{-19} n_{e}+13.34$ in this domain if $T_{e} \geq 30 \mathrm{eV}$. In \#87588, $n_{e}$ ranges from $\approx 3$ to $\approx 3.5 \times 10^{18} \mathrm{~m}^{-3}$ where $\mathrm{S} / \mathrm{XB} \approx 13$ if $T_{e} \geq 30 \mathrm{eV}$. Since $T_{e}$ is around or above $30 \mathrm{eV}$ during inter-ELM for both cases, it can be assumed that it will not be lower during ELMs.

If ELMy ions are essentially reflected as neutrals on the target and promptly re-ionized, the quantitative agreement between LP and calibrated $D_{\alpha}$ signals in Fig. $3 \mathrm{a}$ and $\mathrm{b}$ means that the recycling coefficient $(R)$ during ELMs is near unity. If implantation dominates during ELMs, this match implies that the desorption rate of $D$ neutrals from the near surface reservoir triggered by ELMs equals $\Gamma_{\perp}$ as if $R=1$. This will be discussed in more details in Section 5 below. 
In both eventualities, LP measurements are assumed to be valid during ELMs. However, it could be expected that ELMy electrons would have too much energy to be repelled by biased LP tips, preventing saturation of the ion current. In this case, the LP $\Gamma_{\perp}$ measurements should be significantly underestimated and lower than the calibrated $D_{\alpha}$ signals. The FSM provides an explanation for the validity of LP measurements during ELMs. To preserve quasi-neutrality in ELM filaments while they are transported to the target, electrons must transfer most of their parallel energy to the ions. This occurs very quickly much before the filaments reach the targets if the ELMs are sufficiently compact in the parallel direction, namely $L_{/ /}$>> $L_{E L M}$ [5], which is verified here for both cases in Table 1. The electron perpendicular energy can also be transformed into parallel energy through electron-electron collisions and then transferred to the ions. This process is efficient if the electron collision time $\tau_{\mathrm{e}}$ obtained as follows:

$$
\tau_{e}=3.45 \times 10^{11} \frac{T_{\|}^{3 / 2}}{n_{e} \ln \Lambda} A^{2}\left[-3+(A+3) \frac{\tan ^{-1}\left(A^{1 / 2}\right)}{A^{1 / 2}}\right]^{-1},
$$

with $A=\frac{T_{e}^{p e d}}{T_{\|}}-1$,

the Coulomb Logarithm $\ln \Lambda \approx 15.5$ here,

$$
\text { and } T_{\|}=\frac{T_{e}^{\text {ped }}}{1+\left(0.556 \frac{L_{\|}}{L_{E L M}}\right)^{2}} \text {, }
$$

is such that $\tau_{\mathrm{e}}<<0.556 L / / / c_{s}[5]$. Since $\tau_{\mathrm{e}}$ is not higher than $50 \mu \mathrm{s}$ and $0.556 L / / / c_{s} \sim 1$ $\mathrm{ms}$ in the cases studied here, most of the perpendicular energy of the electrons is transformed into parallel energy and then transferred to the ions.

The near complete transfer of parallel and perpendicular energy by the electrons to the ions during ELMs should allow them to be repelled by LP biasing when they reach the targets. Consequently, saturation of the ion current and $T_{e}$ measurements should be possible during ELMs. These features have been verified here by reconstructing the current-voltage $(I-V)$ characteristic in peak ELM conditions. Assuming that the $I-V$ characteristic is close to:

$I=I_{s a t}\left(1-\mathrm{e}^{\frac{V-V_{f}}{T_{e . E M}}}\right)$,

the saturation current $I_{\text {sat }}$ in A, the floating potential $V_{f}$ in $\mathrm{V}$ and the ELMy electron temperature $T_{e, E L M}$ in $\mathrm{eV}$ can be used as fitting parameters. As shown in Fig. 5, the fit 
gives $T_{e, E L M} \sim 30 \mathrm{eV}$ during the Type-I ELMs of \#84700 and $T_{e, E L M} \sim 40 \mathrm{eV}$ during the Type-III ELMs of \#87588 which is close to the inter-ELM levels, see Fig. 6 . Since $T_{e}$ remains essentially unchanged during ELM and inter-ELM, the assumptions made above about $n_{e} \propto \Gamma_{\perp}$ and $T_{e} \geq 30 \mathrm{eV}$ to estimate $\mathrm{S} / \mathrm{XB}$ are confirmed. The presence of a single saturated $I-V$ characteristic associated with low $T_{e, E L M}$ indicate that the electrons have indeed an energy low enough to allow current saturation of LPs during ELMs and valid $\Gamma_{\perp}$ measurements.

\section{Target ion energy during Type-I and Type-III ELMs}

Since the electron impact energy $E_{e}$ can be neglected, $E_{i}$ in $\mathrm{eV}$ can be calculated such that:

$$
E_{i}+E_{e} \approx E_{i} \approx \frac{q_{\perp}}{\Gamma_{\perp}} .
$$

The time evolution of $E_{i}$ at the strike point is shown in Fig. 3e and $\mathrm{f}$ for cases \#84700 and \#87588. By dividing (2) by (1), the FSM predicts that at peak $q_{\perp}$, the maximum $T_{e}^{\text {ped }}\left(T_{e, \text { max }}^{\text {ped }}\right.$ in $\left.\mathrm{eV}\right)$ and maximum $E_{i}\left(E_{i, \text { max }}\right.$ in $\left.\mathrm{eV}\right)$ are such as: $E_{i, \text { max }} \approx 5.23 T_{e, \text { max }}^{\text {ped }}$. Comparison between $E_{i, \text { max }}$ from Fig. $3 \mathrm{e}$ and $\mathrm{f}$ with coherently averaged $T_{e, \text { max }}^{\text {ped }}$ measurements in Fig. $3 \mathrm{~g}$ and $\mathrm{h}$ indicates that such relation seems verified, as expected.

Coherent averaging of $\Gamma_{\perp}$ and $q_{\perp}$ measurements during Type-I and Type-III ELMs in 72 other cases has been carried out for calculation of $E_{i, \max }$ at peak $q_{\perp}$ and systematic comparison with $T_{e, \text { max }}^{\text {ped }}$. The fit method shown in Fig. 5 has also been applied to the rest of the cases studied here to obtain $T_{e, E L M}$ measurements and see if there is a correlation with $T_{e, \text { max }}^{p e d}$. A very wide range of pedestal conditions has been considered with $T_{e, \text { max }}^{\text {ped }}$ going from $\sim 170 \mathrm{eV}$ to $\sim 1200 \mathrm{eV}$. Fig. 7 shows a very clear linear trend following $y=5.23 x$ for $E_{i, \max }=f\left(T_{e, \max }^{\text {ped }}\right)$ with some acceptable level of uncertainty. Indeed, the deviation $\sigma$ in $\%$ between the ratio $E_{i, \max } / T_{e, \max }^{\text {ped }}$ and 5.23 calculated in Table 2 (see Annex Section) such that:

$$
\sigma=100 \times \frac{\left|\frac{E_{i, \max }}{T_{e, \text { max }}^{p e d}}-5.23\right|}{5.23},
$$

remains mostly around $10 \%$ or below and reaches nearly $30 \%$ only in very few cases. This good agreement confirms that the FSM equations (1) and (2) describe 
appropriately the experimental $\Gamma_{\perp}$ and $q_{\perp}$ time traces in the great variety of conditions of the 74 cases considered here.

In the FSM picture, ELMy ions are essentially kinetic with a dominant parallel motion and the electrons do not have enough energy at the target to establish a sheath with a significant influence [5]. If ELM filaments were made of thermal ions and electrons with $T_{e, E L M} \approx T_{e, \text { max }}^{\text {ped }}$, the electrons could establish a strong sheath with a heat transfer coefficient of $\gamma \approx 8$ [14] and we should have $E_{i, \max } \approx \gamma T_{e, E L M}$. As shown in Fig. 7, the $T_{e, E L M}$ measurements are far too low to explain the very high $E_{i, \max }$ observed in experiments. Therefore, sheath effects can be ignored during ELMs, as predicted by the FSM.

\section{Discussion on energy reflections and deposition during ELMs}

According to the TRIM database [15], the energy and particle reflection coefficients for $\mathrm{D}$ ions striking a smooth $\mathrm{W}$ surface with $E_{i}$ in the range $1-10 \mathrm{keV}$ are between 0.9 and 0.7. Consequently, most of impinging ELMy ions in the 74 cases studied here could be reflected as fast neutrals at the target and spread their energy over a wide area of the divertor without depositing significant amounts at the strike point. Therefore, $E_{i, \max }$ calculated from IR and LP measurements in this region should not be higher than a few hundreds of $\mathrm{eV}$. This is in contradiction with $E_{i, \text { max }}$ ranging from $\sim 1$ to nearly $\sim 7 \mathrm{keV}$ in Fig. 7, consistently with FSM predictions. Moreover, ELM energy depositions of the order of $\sim \Delta W$ on less than $\sim 50 \%$ of Tile 5 area (Fig. 2) are routinely found by IR measurements in JET-ILW [16,17]. This suggests that a large fraction of the ELM energy is indeed deposited on a limited area of the W PFC.

The reflection of high energy $\mathrm{D}$ particles (ions or neutrals) on $\mathrm{W}$ with shallow angle of incidence should be nearly specular [18]. After their first reflection, fast ELMy neutrals with a dominant toroidal motion and $~ 70-90 \%$ of their initial ion energy can interact with the dense ELMy ion flow through charge exchange $(\mathrm{CX})$, electron impact ionization and ion impact ionization. The mean free path deduced from ADAS [13] for a $D$ fast neutral before the occurrence of a CX reaction is of the order of a few $\mathrm{cm}$ for ion densities $n_{i} \approx n_{e} \sim 10^{20} \mathrm{~m}^{-3}$ in the domain of energies $1-10$ $\mathrm{keV}$. On the other hand, electron impact ionization has a strong effect only at low energy and ion impact ionization is negligible. Therefore, most reflected fast neutrals can quickly become ions again through $\mathrm{CX}$ and come back to the target by following the magnetic field lines to leave more of their energy, see Fig. 8. ELMy particles could thus bounce back and forth between the target and the plasma and deposit their energy in the PFC by successive impacts and progressive implantations.

The remaining total energy of a fast ion population after a given number of reflections on $\mathrm{W}$ has been assessed with the SRIM code [19] using a binary collision approximation and the TRIM database [15]. For ions with an initial $E_{i}=5 \mathrm{keV}$ and an angle of incidence of $5^{\circ}$ with a smooth W surface, most of the total initial energy is deposited on the target after $5-6$ reflections, see Fig. 9a. If surface roughness is considered, collisions with irregularities with a surface incidence angle up to $90^{\circ}$ are 
possible. In this extreme case, most of the energy is deposited on the target after only $1-2$ reflections, see Fig. $9 \mathrm{~b}$.

Observation of strong desorption associated with ELMs in JET-ILW [20-22] confirms that ELMy particles dominantly end up implanted after a few reflections. According to SRIM calculations [19], multi-keV D ions with shallow angles of incidence have an implantation depth of a few tens of $\mathrm{nm}$ in W. During ELMs, surface temperatures above $1000^{\circ} \mathrm{C}$ are measured by IR in JET-ILW and in these conditions, the mobility of implanted ELMy particles back to the surface should be fast with a diffusion time on the $\mu \mathrm{s}$ scale [23]. Therefore, the implantation of ELMy particles and the release of their energy should trigger the quasi-simultaneous fast diffusion and desorption of low energy $D_{2}$ molecules from the surface. The match between $\Gamma_{\perp}$ from LPs and the ionization rate density from calibrated $D_{\alpha}$ signals (Fig.

$3 a$ and $b$ ) implies that the implantation of a given amount of ELMy particles releases an equal amount of $D$ neutrals. This recycling process is consistent with a saturated near surface reservoir expected with W PFCs [24].

Since the $C X$ reaction conserves the charge, the initial charge carried by the ELMy ions leaving the pedestal should be the same as the charge carried by the fast ions impacting the target. Thus, $\Gamma_{\perp}$ measurements by LPs should not be affected by the multiple particle reflections and the slow ions produced after dissociation of $D_{2}$ molecules and electron ionization (Fig. 8) should be the main contributors to the background $\Gamma_{0}$ discussed in Section 2.

Since ELMy particle are very fast, the succession of impacts and CX reactions followed by implantation should not take more than a few $\mu \mathrm{s}$. This is nearly instantaneous compared to the ELM duration which is usually of the order of a ms or more, see Fig. 3.

Quantitative description of the energy deposition mechanism on W during ELMs would require kinetic modelling of ELMy ions and neutrals accounting for the dominant atomic physics processes occurring in the plasma as well as the target properties. Such work is beyond the scope of this paper and should be the object of further studies.

\section{Conclusions}

Validation of the predictions of the "Free-Streaming" model (FSM) for 74 Type-I and Type-III ELMy H-mode experiments with a very wide range of conditions has been carried out with success in JET-ITER-Like Wall (ILW) and presented here.

It has been possible to fit the experimental time evolution of ELM power and particle flux densities at the outer target strike point in examples of Type-I and TypeIII ELMy H-mode discharges in JET-ILW with the FSM. The parallel connection length and ELM length used as fitting parameters are consistent with the experimental ELM energy and with ELM modelling using the JOREK code $[11,12]$. 
FSM prediction of low target electron energy during ELMs due to near complete transfer of energy from electrons to ions to conserve quasi-neutrality has been verified in all Type-I and Type-III ELMy $\mathrm{H}$-modes analysed here.

The ion impact energy at the strike point at peak power density $\left(E_{i, \max }\right)$ predicted by the FSM during ELMs matches the experimental estimates calculated from divertor infrared thermography (IR) and Langmuir probe measurements. As expected from the model, $E_{i, \max }$ is proportional to the pedestal temperature before the ELM crash with a factor 5.23 and ranges from $1 \mathrm{keV}$ to nearly $7 \mathrm{keV}$ in the 74 cases studied here.

Tungsten is known to reflect very efficiently energetic ions at shallow angles of incidence [15] which suggests that energy deposition during ELMs should be spread on a very wide area of the divertor. In this picture, $E_{i, \max }$ estimates at the strike-point should not exceed a few hundreds of eV. This is generally inconsistent with ELM energy measurements from IR of the order of the variation of the stored energy on a limited area of JET-ILW divertor targets $[16,17]$.

Charge exchange could force ELMy particles to bounce back and forth between the plasma and the target to allow efficient energy deposition by successive impacts and progressive implantations. Just a few reflections may be sufficient if surface roughness is considered. Full description of this mechanism would require kinetic modelling of ELMy ions and neutrals accounting for the atomic physics processes occurring in the plasma as well as the target properties. Such work is beyond the scope of this paper and should be the object of further studies.

\section{Acknowledgments}

This work has been carried out within the framework of the EUROfusion Consortium and has received funding from the Euratom research and training programme 20142018 under grant agreement No 633053. IST activities also received financial support from "Fundação para a Ciência e Tecnologia" through project UID/FIS/50010/2013. The views and opinions expressed herein do not necessarily reflect those of the IST or of the European Commission.

\section{Annex}

(Insert Table 2 with captions)

\section{References}

[1] J.P. Gunn et al., Nuclear Materials and Energy (2016), http://dx.doi.org/10.1016/i.nme.2016.10.005

[2] C. Guillemaut et al., Phys. Scr. T167 (2016) 014005

[3] W. Fundamenski et al., Plasma Phys. Control. Fusion 48 (2006) 109-156

[4] T. Eich et al., J. Nucl. Mater. 390-391 (2009) 760-763

[5] D. Moulton et al., Plasma Phys. Control. Fusion 55 (2013) 085003

[6] C. Guillemaut et al., Plasma Phys. Control. Fusion 57 (2015) 085006

[7] R.A. Pitts et al., Nucl. Fusion 43 (2003) 1145-1166 
[8] R.A. Pitts et al., Nucl. Fusion 46 (2006) 82

[9] M.W. Jakubowski et al., J. Nucl. Mater. 390-391 (2009) 781

[10] A. Kirk et al., J. Phys. Conf. Ser. 123 (2008) 012011

[11] S. Pamela et al., Plasma Phys. Control. Fusion 58 (2016) 014026

[12] G.T.A. Huysmans et al., Plasma Phys. Control. Fusion 51 (2009) 124012

[13] H.P. Summers. Atomic data and analysis structure user manual. University of Strathclyde, 2nd edition, 2004

[14] S. Marsen et al., J. Nucl. Mat. 438 (2013) 393-396

[15] W. Eckstein et al, 2002 Report IPP 9/132

[16] A.R. Field et al., Plasma Phys. Control. Fusion 59 (2017) 095003

[17] Private communication with L. Horvath

[18] W. Eckstein and D.B. Heifetz, J. Nucl. Mat. 145-147 (1987) 332

[19] J.F. Ziegler, Nucl. Instr. Meth. B 268 (2010) 1818

[20] S. Wiesen et al., Nucl. Fusion 57 (2017) 066024

[21] S. Brezinsek et al., Phys. Scr. T167 (2016) 014076

[22] Private communication with K. Heinola

[23] K. Heinola et al., Physical Review B 81 (2010) 073409

[24] S. Brezinsek et al., J. Nucl. Mat. 463 (2015) 11-21 


\section{Figure captions :}

Fig. 1 Range of power and ELM frequency in the Type-I or Type-III H-mode discharges studied here. These cases involved hydrogen or deuterium as main species.

Fig. 2 Magnetic equilibria for the Type-I (\#84700) and Type-III (\#87588) ELMy Hmode plasmas, diagnostics involved and tile numbers.

Fig. 3 Examples of coherently averaged (a,b) $\Gamma_{\perp}$ from LPs (blue dots) and calibrated $\mathrm{D}_{\alpha}$ (magenta squares), (c,d) $q_{\perp}$ (red dots), (e,f) $E_{i}$ (green dots) and (g,h) $T_{e}^{\text {ped }}$ (black dots) time traces for Type-I and Type-III ELMs in discharges \#84700 and \#87588 respectively. All target measurements are made at the OT strike-point. To facilitate the comparison with LPs, $\Gamma_{\perp}$ obtained from calibrated $D_{\alpha}$ is also in kA.m ${ }^{-2}$. The dashed black curves in (a-c) are the FSM fits.

Fig. $4 n_{e}$ and $T_{e}$ dependence of S/XB from ADAS [13].

Fig. 5 Example of $I-V$ characteristic reconstructions (blues dots) and fits (red curves) during Type-I and Type-III ELMs in \#84700 (left) and \#87588 (right) respectively. About 100 ELMs were used in both cases for such reconstructions.

Fig. $6 T_{e}$ measurements obtained from I-V characteristic fit during inter-ELM for discharges \#84700 (left) and \#87588 (right). The ELM starts after $\mathrm{t}=1 \mathrm{~ms}$ in \#84700 and after $\mathrm{t}=0.2 \mathrm{~ms}$ in \#87588.

Fig. 7 Linear dependence between $E_{i, \text { max }}$ and $T_{e, \text { max }}^{\text {ped }}$ during ELMs (red and blue bullets). No dependence can be found between $T_{e, E L M}$ and $T_{e}^{\text {ped }}$ (red and blue squares). Red points correspond to Type-I ELMs experimental data and blue points to Type-III ELMs. The black line equation is $y=5.23 x$.

Fig. 8 Examples of atomic physics processes occurring at the target during ELMs.

Fig. 9 Remaining total energy of a fast ion population after a given number of reflections on W calculated with the SRIM code [19] for (a) $5^{\circ}$ and (b) $90^{\circ}$ surface incidence angles. The total reflected energy is normalized to the total initial energy.

\section{Table captions:}

Table 1 Experimental and fitting parameters for Type-I ELMs in \#84700 and Type-III ELMs in \#87588

Table 2 Main parameters for each of the 74 cases at the specific times used for the present study 


\begin{tabular}{|c|c|c|c|c|}
\hline & $T_{e}^{\text {ped }}(\mathrm{eV})$ & $n_{e}^{\text {ped }}\left(\mathrm{m}^{-3}\right)$ & $L_{/ /}(\mathrm{m})$ & $L_{E L M}(\mathrm{~m})$ \\
\hline$\# 84700$ & 880 & $3 \times 10^{19}$ & 864 & 210 \\
\hline$\# 87588$ & 550 & $1.3 \times 10^{19}$ & 112 & 5 \\
\hline
\end{tabular}

Table 1 


\begin{tabular}{|c|c|c|c|c|c|c|c|c|c|c|c|c|c|}
\hline Shot & Time (s) & Spec. & Imp. & $\begin{array}{l}\text { ELM } \\
\text { type }\end{array}$ & $\begin{array}{c}f_{E L M} \\
(\mathrm{~Hz})\end{array}$ & $\begin{array}{l}P_{\text {in }} \\
\text { (MW) }\end{array}$ & $\begin{array}{l}I_{P} \\
(\mathrm{MA})\end{array}$ & $\begin{array}{l}B_{T} \\
(\mathrm{~T})\end{array}$ & $\begin{array}{l}T_{e, E L M} \\
(\mathrm{eV})\end{array}$ & $\begin{array}{l}E_{i, \max } \\
(\mathrm{eV})\end{array}$ & $\begin{array}{l}T_{e, \max }^{\text {ped }} \\
(\mathrm{eV})\end{array}$ & Ratio & $\begin{array}{l}\text { Dev. } \\
\text { (\%) }\end{array}$ \\
\hline 84580 & $16.4-17.9$ & D & & 1 & 25 & 3.8 & 2.0 & 2.1 & 55 & 1387 & 310 & 4.5 & 14.5 \\
\hline 84584 & $9-11.5$ & $\mathrm{D}$ & & 1 & 14 & 10 & 2.0 & 2.1 & 32 & 3436 & 615 & 5.6 & 6.8 \\
\hline 84587 & $10-12$ & $\mathrm{D}$ & & 1 & 110 & 14 & 2.0 & 2.1 & 11 & 1097 & 295 & 3.7 & 28.9 \\
\hline 84589 & $12-14$ & $\mathrm{D}$ & & 1 & 50 & 13 & 2.0 & 2.1 & 10 & 3436 & 650 & 5.3 & 1.1 \\
\hline 84613 & $13-15$ & $\mathrm{D}$ & & 1 & 57 & 12.5 & 2.0 & 2.1 & 18 & 3105 & 570 & 5.4 & 4.2 \\
\hline 84614 & $13.15-13.5$ & $\mathrm{D}$ & & 1 & 55 & 12.5 & 2.0 & 2.1 & 13 & 2652 & 477 & 5.6 & 6.3 \\
\hline 84623 & $6.2-7.4$ & $\mathrm{D}$ & & 1 & 125 & 19.2 & 1.7 & 2.0 & 28 & 3125 & 798 & 3.9 & 25.1 \\
\hline 84635 & $6.2-7.8$ & $\mathrm{D}$ & & 1 & 110 & 13 & 1.7 & 2.0 & 25 & 2592 & 641 & 4.0 & 22.7 \\
\hline 84678 & $9.8-11.1$ & $\mathrm{D}$ & & 1 & 100 & 14 & 2.0 & 2.1 & 23 & 3635 & 695 & 5.2 & 0.0 \\
\hline 84682 & $6-6.7$ & $\mathrm{D}$ & & 1 & 60 & 21 & 2.1 & 2.0 & 32 & 5659 & 1165 & 4.9 & 7.1 \\
\hline 84687 & $12-14$ & $\mathrm{D}$ & & 1 & 105 & 17 & 2.5 & 2.4 & 21 & 2825 & 495 & 5.7 & 9.1 \\
\hline 84689 & $9-11.5$ & $\mathrm{D}$ & & 1 & 20 & 11 & 2.0 & 2.1 & 18 & 4364 & 763 & 5.7 & 9.4 \\
\hline 84693 & $9-11.5$ & $\mathrm{D}$ & & 1 & 25 & 11 & 2.0 & 2.1 & 15 & 4025 & 722 & 5.6 & 6.6 \\
\hline 84694 & $12.5-14.6$ & $\mathrm{D}$ & & 1 & 25 & 11 & 2.0 & 2.1 & 12 & 3169 & 598 & 5.3 & 1.3 \\
\hline 84700 & $6-7.5$ & $\mathrm{D}$ & & 1 & 50 & 15 & 2.0 & 2.3 & 29 & 5180 & 880 & 5.9 & 12.5 \\
\hline 84718 & $13-14.5$ & $\mathrm{D}$ & & 1 & 100 & 20 & 2.7 & 2.6 & 23 & 2661 & 547 & 4.9 & 7.0 \\
\hline 84719 & $13.2-13.8$ & $\mathrm{D}$ & & 1 & 120 & 24 & 3.0 & 2.9 & 22 & 3447 & 690 & 5.0 & 4.5 \\
\hline 84720 & $12.3-13.2$ & $\mathrm{D}$ & & 1 & 85 & 23 & 3.0 & 2.9 & 8 & 3385 & 704 & 4.8 & 8.1 \\
\hline 84721 & $12.5-13.5$ & $\mathrm{D}$ & & 1 & 101 & 20 & 3.0 & 2.9 & 7 & 3650 & 690 & 5.3 & 1.1 \\
\hline 84722 & $13.2-13.8$ & $\mathrm{D}$ & & 1 & 127 & 23.5 & 3.0 & 2.9 & 20 & 3209 & 660 & 4.9 & 7.0 \\
\hline 84724 & $12.8-13.8$ & $\mathrm{D}$ & & 1 & 37 & 22 & 3.0 & 2.9 & 21 & 4480 & 833 & 5.4 & 2.8 \\
\hline 84759 & $9-9.9$ & $\mathrm{D}$ & & 1 & 32 & 17 & 2.5 & 2.7 & 11 & 4252 & 806 & 5.3 & 0.9 \\
\hline 84760 & $10.6-11.4$ & $D$ & & 1 & 100 & 21.7 & 2.5 & 2.7 & 17 & 4292 & 780 & 5.5 & 5.2 \\
\hline 84761 & $9.5-10.5$ & $\mathrm{D}$ & & 1 & 16 & 17.3 & 2.5 & 2.7 & 13 & 4806 & 910 & 5.3 & 1.0 \\
\hline 84772 & $9.5-10.5$ & $\mathrm{D}$ & & 1 & 58 & 16.5 & 2.5 & 2.7 & 10 & 3664 & 715 & 5.1 & 2.0 \\
\hline 84778 & $12.6-14.4$ & $\mathrm{D}$ & & 1 & 35 & 24 & 3.0 & 2.9 & 26 & 4864 & 960 & 5.1 & 3.1 \\
\hline 84782 & $12.6-14.4$ & $\mathrm{D}$ & & 1 & 38 & 24 & 3.0 & 2.9 & 25 & 5550 & 1053 & 5.3 & 0.8 \\
\hline 84794 & $4.5-6$ & $\mathrm{D}$ & & 1 & 30 & 17.7 & 1.4 & 1.7 & 30 & 6362 & 1172 & 5.4 & 3.8 \\
\hline 84798 & $4.5-6$ & $\mathrm{D}$ & & 1 & 40 & 6.3 & 1.4 & 1.7 & 27 & 3330 & 560 & 5.9 & 13.7 \\
\hline 85026 & $11.4-12.9$ & $\mathrm{D}$ & & 1 & 50 & 16 & 2.0 & 2.0 & 18 & 2755 & 564 & 4.9 & 6.6 \\
\hline 85032 & $11.4-12.9$ & $\mathrm{D}$ & & 1 & 44 & 13.6 & 2.0 & 2.0 & 15 & 2940 & 530 & 5.5 & 6.1 \\
\hline 85041 & $12-14$ & $\mathrm{D}$ & & 1 & 16 & 10.8 & 2.0 & 2.1 & 25 & 3610 & 684 & 5.3 & 0.9 \\
\hline 85043 & $12-14$ & $\mathrm{D}$ & & 1 & 18 & 11 & 2.0 & 2.1 & 17 & 2598 & 580 & 4.5 & 14.4 \\
\hline 85046 & $12-14$ & $\mathrm{D}$ & & 1 & 19 & 11 & 2.0 & 2.1 & 24 & 2338 & 520 & 4.5 & 14.0 \\
\hline 87517 & $15.5-17$ & $\mathrm{D}$ & & 1 & 120 & 9.5 & 1.2 & 1.8 & 26 & 2300 & 560 & 4.1 & 21.5 \\
\hline 87587 & $14.2-15.4$ & $\mathrm{D}$ & & 1 & 100 & 5.7 & 2.0 & 2.4 & 28 & 1550 & 350 & 4.4 & 15.3 \\
\hline 87874 & $19-21$ & $\mathrm{D}$ & & 1 & 50 & 6.8 & 1.7 & 1.8 & 25 & 892 & 240 & 3.7 & 28.9 \\
\hline 89426 & $12.4-14.5$ & $\mathrm{D}$ & & 1 & 39 & 13 & 2.0 & 2.1 & 19 & 3640 & 562 & 6.5 & 23.8 \\
\hline 89708 & $12-13.6$ & $\mathrm{D}$ & & 1 & 35 & 19.6 & 2.5 & 2.6 & 30 & 1911 & 408 & 4.7 & 10.4 \\
\hline
\end{tabular}




\begin{tabular}{|c|c|c|c|c|c|c|c|c|c|c|c|c|c|}
\hline 89709 & $11-13$ & $\mathrm{D}$ & & 1 & 45 & 19.5 & 2.5 & 2.6 & 29 & 2150 & 433 & 5.0 & 5.1 \\
\hline 90405 & $5-6.5$ & $\mathrm{D}$ & & 1 & 95 & 10.5 & 1.4 & 1.7 & 32 & 1870 & 355 & 5.3 & 0.7 \\
\hline 90406 & $5-7.5$ & D & & 1 & 70 & 10.5 & 1.4 & 1.7 & 37 & 2180 & 412 & 5.3 & 1.2 \\
\hline 90580 & $14-16$ & D & & 1 & 90 & 10 & 1.7 & 2.3 & 56 & 1489 & 310 & 4.8 & 8.2 \\
\hline 90725 & $15.8-17$ & D & $\mathrm{N}$ & 1 & 100 & 19.6 & 2.0 & 2.3 & 55 & 3333 & 650 & 5.1 & 2.0 \\
\hline 90997 & $21.6-22.9$ & $\mathrm{H}$ & & 1 & 23 & 8.5 & 1.7 & 1.8 & 29 & 825 & 168 & 4.9 & 6.1 \\
\hline 90998 & $19-21$ & $\mathrm{H}$ & & 1 & 80 & 7.3 & 1.7 & 1.8 & 24 & 783 & 158 & 5.0 & 5.2 \\
\hline 91112 & $16-17$ & $\mathrm{H}$ & & 1 & 110 & 6.8 & 1.7 & 1.8 & 25 & 1186 & 240 & 4.9 & 5.5 \\
\hline 91115 & $17-20$ & $\mathrm{H}$ & & 1 & 52 & 7.7 & 1.7 & 1.8 & 35 & 1089 & 230 & 4.7 & 9.5 \\
\hline 91466 & $17-18$ & $\mathrm{H}$ & & 1 & 80 & 9 & 1.7 & 1.8 & 46 & 1727 & 329 & 5.2 & 0.4 \\
\hline 91597 & $16.5-17.1$ & $\mathrm{H}$ & & 1 & 70 & 14 & 1.7 & 1.8 & 53 & 2510 & 450 & 5.6 & 6.6 \\
\hline 91599 & $16.5-17.1$ & $\mathrm{H}$ & & 1 & 60 & 12.8 & 1.7 & 1.8 & 53 & 2884 & 505 & 5.7 & 9.2 \\
\hline 91603 & $16-17$ & $\mathrm{H}$ & & 1 & 56 & 14.5 & 1.7 & 1.8 & 40 & 3360 & 540 & 6.2 & 19.0 \\
\hline 91605 & $14-14.5$ & $\mathrm{H}$ & & 1 & 70 & 8.8 & 1.7 & 1.8 & 42 & 1513 & 293 & 5.2 & 1.3 \\
\hline 91606 & $15.35-15.85$ & $\mathrm{H}$ & & 1 & 60 & 14 & 1.7 & 1.8 & 49 & 2920 & 510 & 5.7 & 9.5 \\
\hline 91610 & $14.5-16$ & $\mathrm{H}$ & & 1 & 80 & 11.5 & 1.7 & 1.7 & 9 & 2158 & 434 & 5.0 & 4.9 \\
\hline 91611 & $14.2-15$ & $\mathrm{H}$ & & 1 & 55 & 17 & 1.7 & 1.7 & 8 & 2980 & 513 & 5.8 & 11.1 \\
\hline 91725 & $13.2-14.05$ & $\mathrm{H}$ & & 1 & 50 & 8.1 & 1.7 & 1.8 & 58 & 927 & 210 & 4.4 & 15.6 \\
\hline 91962 & $12-13.4$ & $\mathrm{D}$ & & 1 & 25 & 26.6 & 3.2 & 3.0 & 47 & 3572 & 716 & 5.0 & 4.6 \\
\hline 92130 & $8.5-9.5$ & D & $\mathrm{N}$ & 1 & 110 & 20.6 & 2.0 & 2.4 & 9 & 4558 & 850 & 5.4 & 2.5 \\
\hline 92135 & $8.5-9.5$ & D & $\mathrm{N}$ & 1 & 50 & 20 & 2.0 & 2.4 & 6 & 4402 & 820 & 5.4 & 2.6 \\
\hline 92141 & $8.3-9.2$ & $\mathrm{D}$ & $\mathrm{N}$ & 1 & 60 & 18.5 & 2.0 & 2.4 & 9 & 3859 & 770 & 5.0 & 4.2 \\
\hline 92332 & $12.4-13.6$ & $\mathrm{D}$ & & 1 & 44 & 25.7 & 3.2 & 3.0 & 45 & 3357 & 625 & 5.4 & 2.7 \\
\hline 92335 & $12.4-13.6$ & $\mathrm{D}$ & & 1 & 40 & 24.8 & 3.2 & 3.0 & 39 & 2734 & 550 & 5.0 & 5.0 \\
\hline 81878 & $20.2-21$ & $\mathrm{D}$ & & 3 & 400 & 4.4 & 2.0 & 2.4 & 30 & 2554 & 475 & 5.4 & 2.8 \\
\hline 81881 & $17.9-18.9$ & $\mathrm{D}$ & & 3 & 1330 & 6.3 & 2.0 & 2.4 & 33 & 2787 & 478 & 5.8 & 11.5 \\
\hline 81883 & $21.2-22.3$ & D & & 3 & 420 & 5.2 & 2.0 & 2.4 & 32 & 2498 & 520 & 4.8 & 8.1 \\
\hline 87587 & $20.1-20.7$ & $D$ & & 3 & 270 & 5.7 & 2.0 & 2.4 & 49 & 2530 & 580 & 4.4 & 16.6 \\
\hline 87588 & $20.44-20.54$ & D & & 3 & 1200 & 5.6 & 2.0 & 2.4 & 41 & 2626 & 550 & 4.8 & 8.7 \\
\hline 91411 & $9-11$ & $\mathrm{H}$ & $\mathrm{Ne}$ & 3 & 210 & 9.5 & 1.0 & 1.0 & 32 & 1080 & 200 & 5.4 & 3.3 \\
\hline 91419 & $9-11$ & $\mathrm{H}$ & & 3 & 200 & 8 & 1.0 & 1.0 & 28 & 853 & 160 & 5.3 & 1.9 \\
\hline 91432 & $9-11$ & $\mathrm{H}$ & & 3 & 220 & 10.4 & 1.0 & 1.0 & 37 & 825 & 175 & 4.7 & 9.9 \\
\hline 91539 & $8-10$ & $\mathrm{H}$ & & 3 & 175 & 6.3 & 1.0 & 1.0 & 50 & 859 & 150 & 5.7 & 9.5 \\
\hline 91595 & $15.4-15.9$ & $\mathrm{H}$ & & 3 & 350 & 10.4 & 1.7 & 1.8 & 40 & 2462 & 520 & 4.7 & 9.5 \\
\hline 91597 & $15.15-15.6$ & $\mathrm{H}$ & & 3 & 140 & 12.7 & 1.7 & 1.8 & 59 & 1488 & 305 & 4.9 & 6.7 \\
\hline 91666 & $15.4-16.4$ & $\mathrm{H}$ & & 3 & 700 & 13 & 1.7 & 1.8 & 59 & 3190 & 602 & 5.3 & 1.3 \\
\hline
\end{tabular}

Table 2 


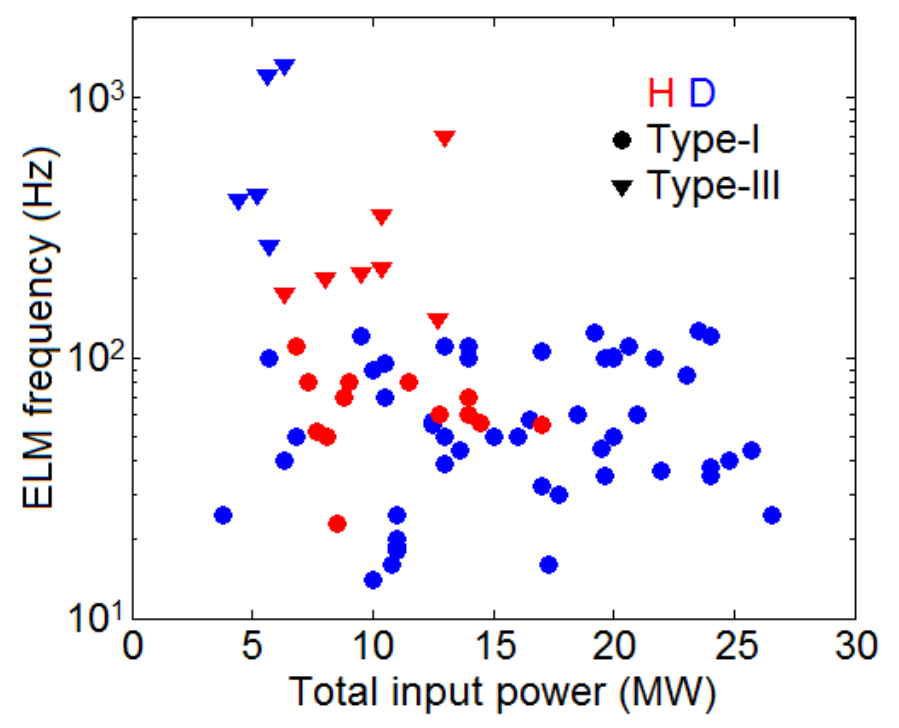

Fig. 1 

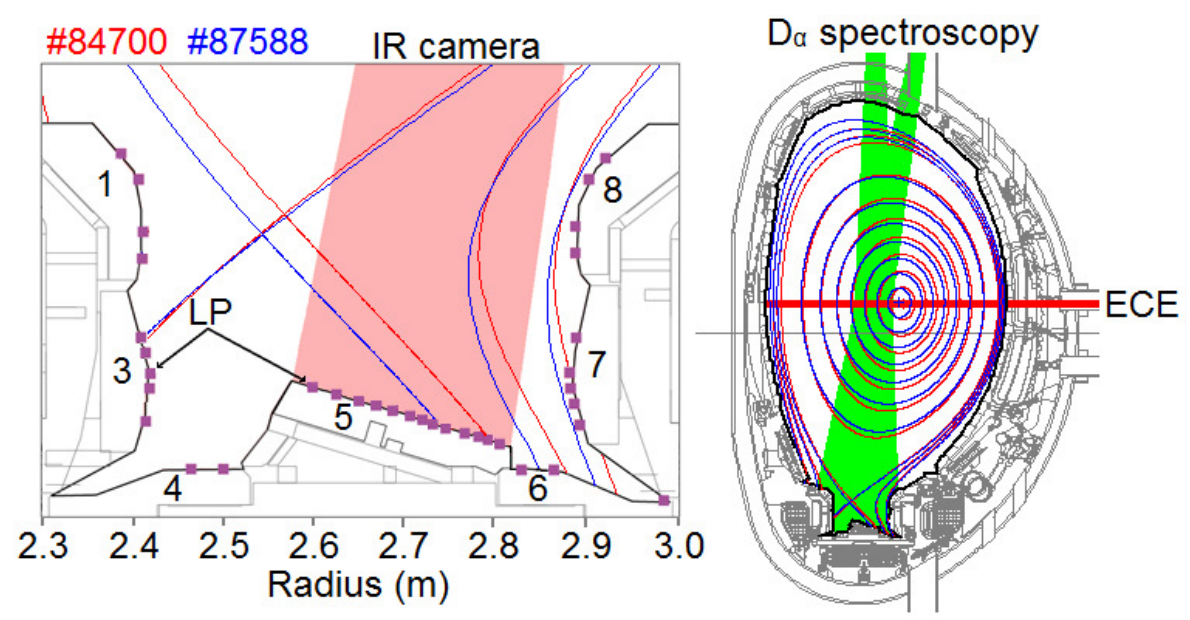

Fig. 2 

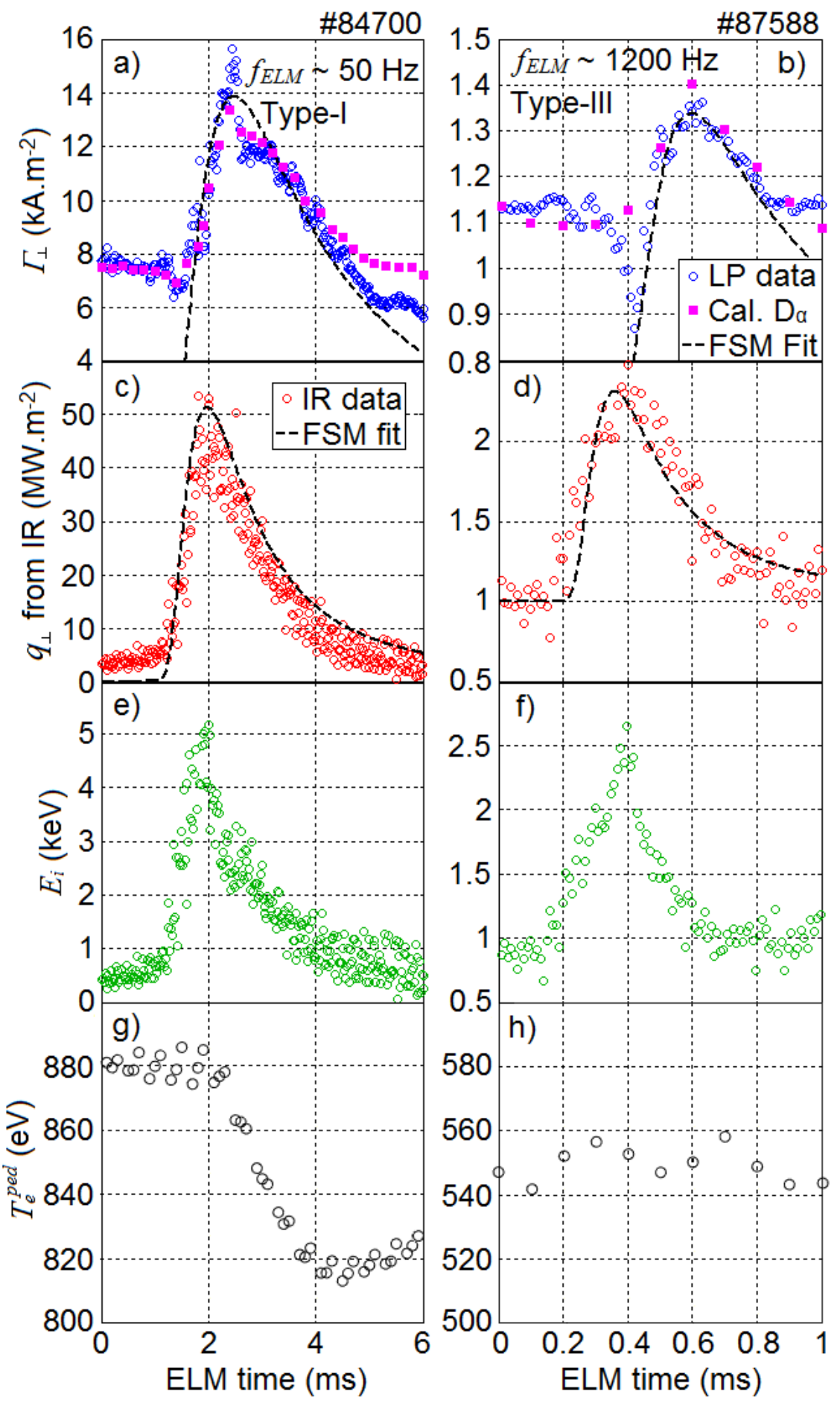

Fig. 3 


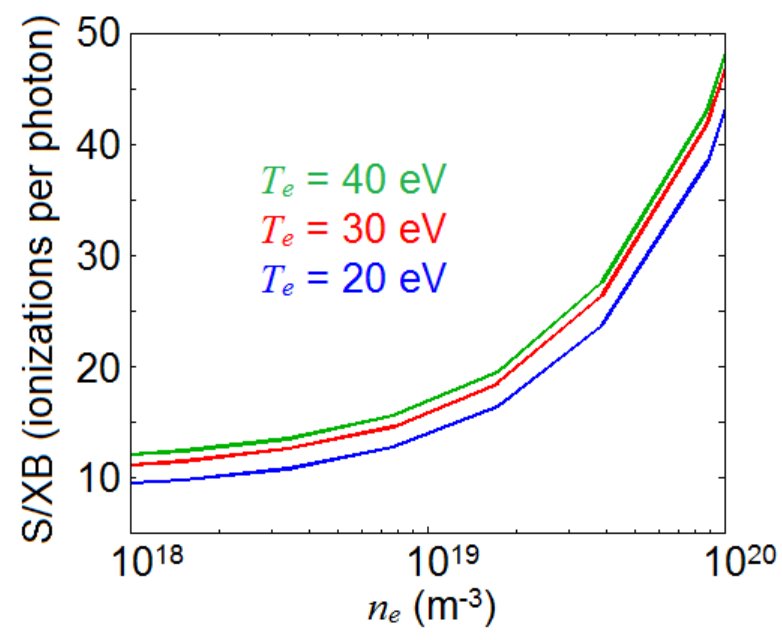

Fig. 4 

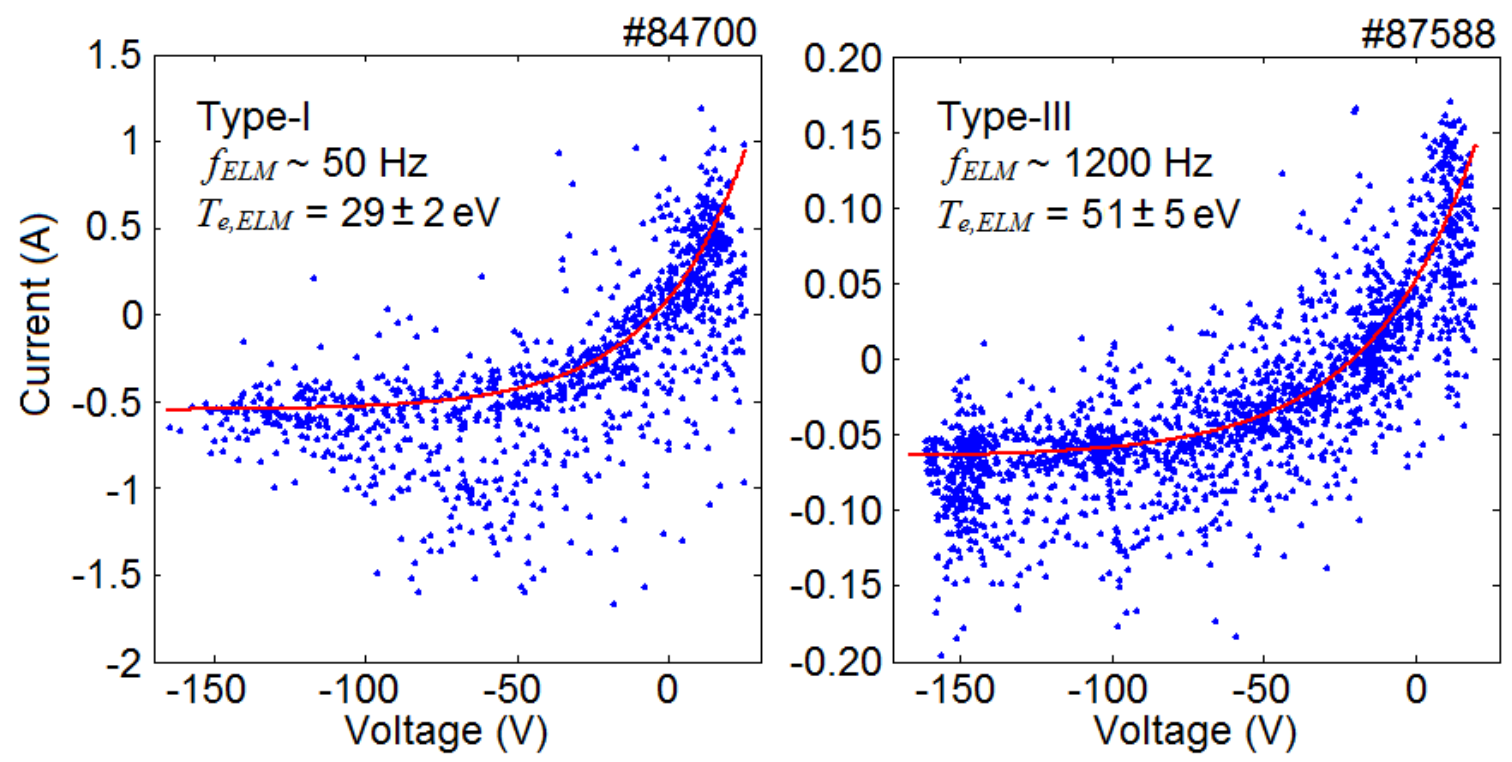

Fig. 5 


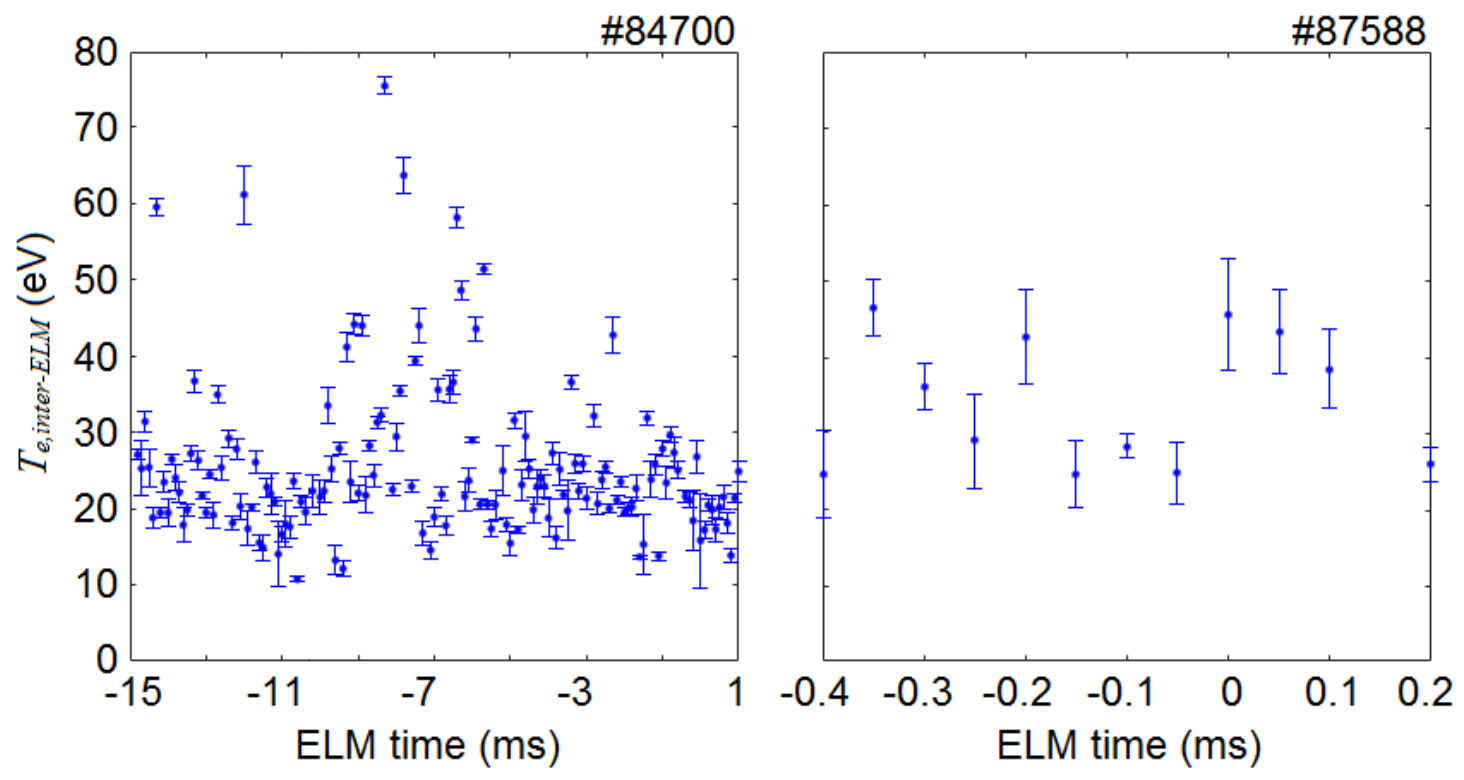

Fig. 6 


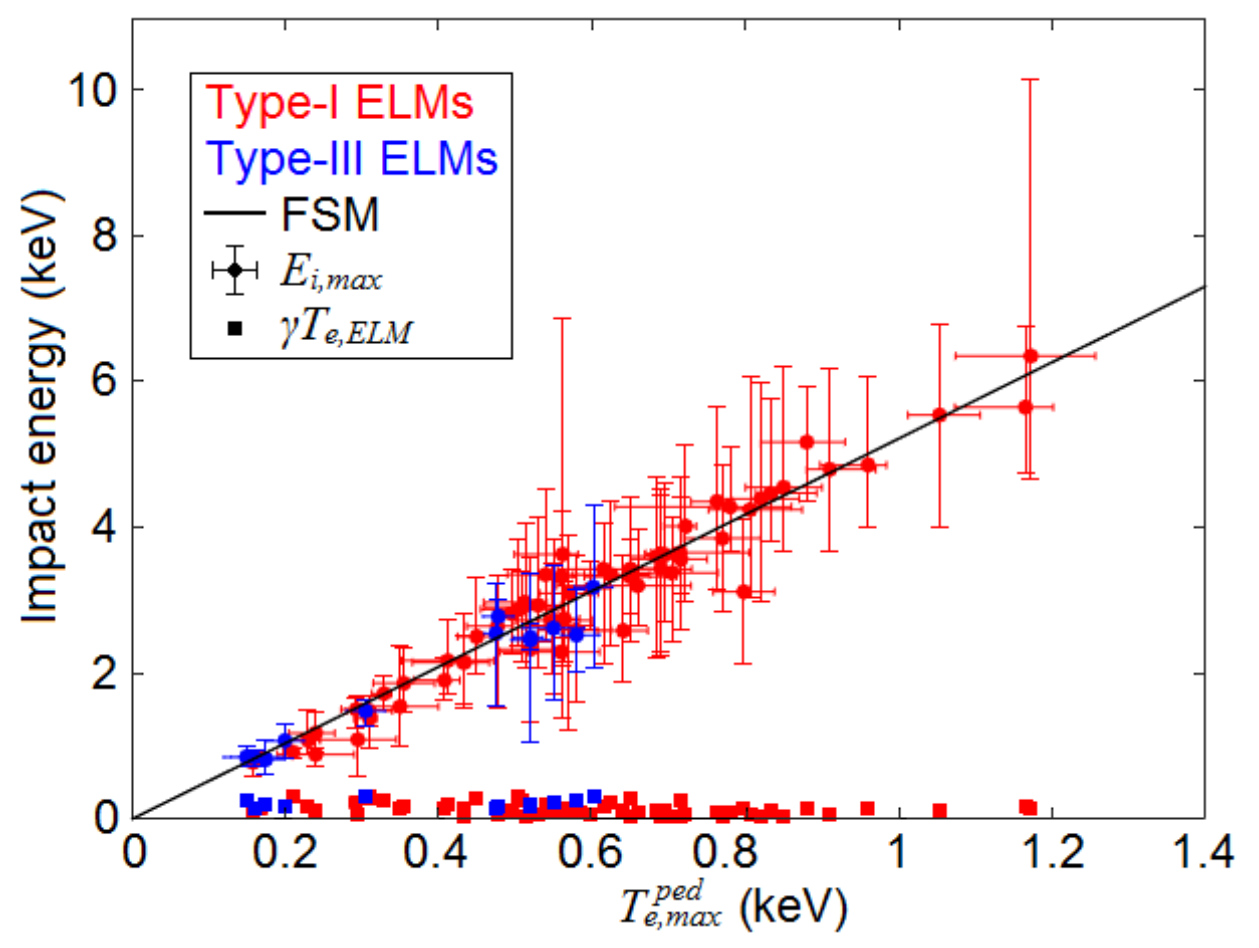

Fig. 7 


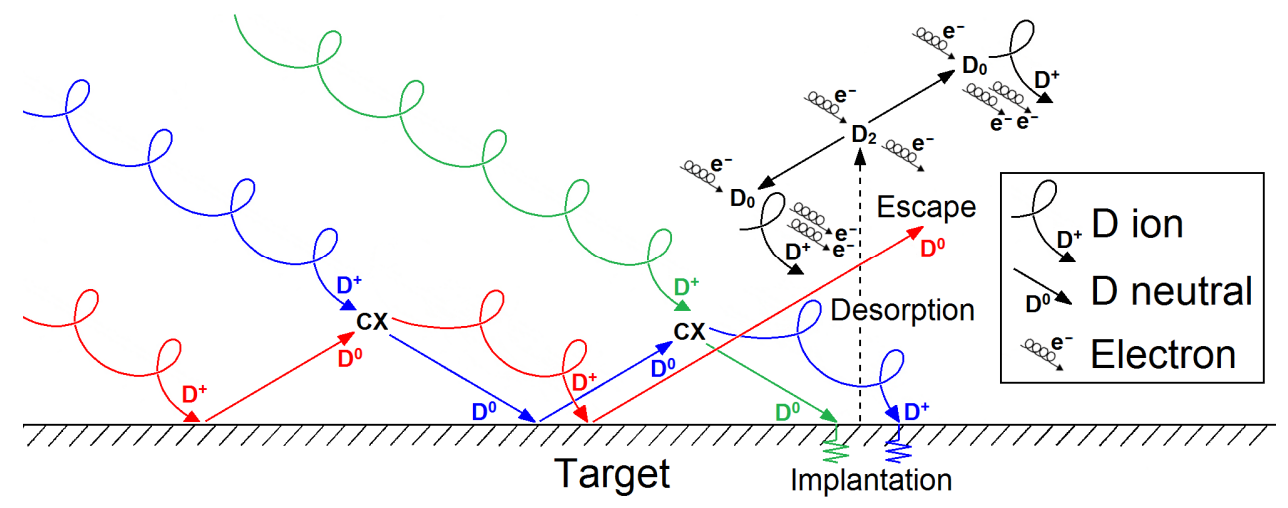

Fig. 8 


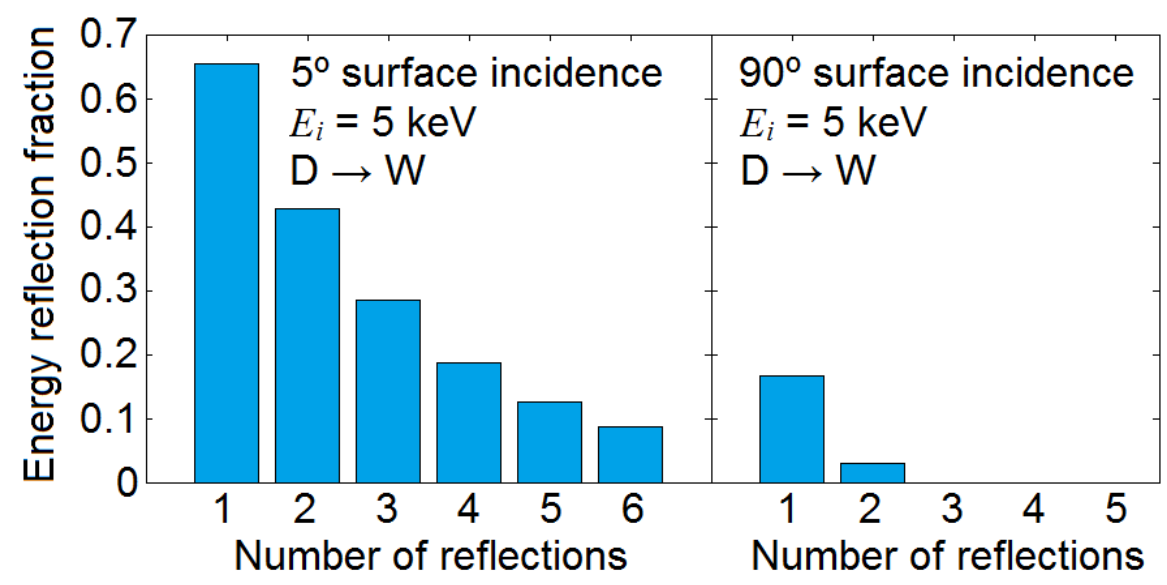

Fig. 9 